\title{
Multi label classification on multi resident in smart home using classifier chains
}

\begin{abstract}
Rapid development in smart home environment are driven by the development of computing and sensing technology, has been changing the landscape of home residentôs daily life. Among others, activity recognition has become an interesting area of exploration in the domain of smart home. Activity recognition describes the paradigm of obtaining raw sensor data as inputs and predicting a home residentôs activity accordingly consist from environmental-based sensors that are embedded into the environment. The recognized patterns are based on Activity of Daily Living (ADL). In this paper, we design a multi label classification framework to cater multi resident in smart home environment using Classifier Chains approach. Human activities, everyday are gradually becoming complex especially relating with multi resident requirement and thus complicate the inferences of activities in smart home. Hence, this paper will highlight the methodology of sensing technology involved as well as important research works done in activity recognition area specifically on multi resident complex activity recognition involving interaction activity of multi resident within the same environment. Furthermore, this paper also discussed potential directions for future research in the activity recognition.
\end{abstract}

Keyword: Classifier chains; Complex activity recognition; Multi label classification; Multi resident; Smart home sensor 